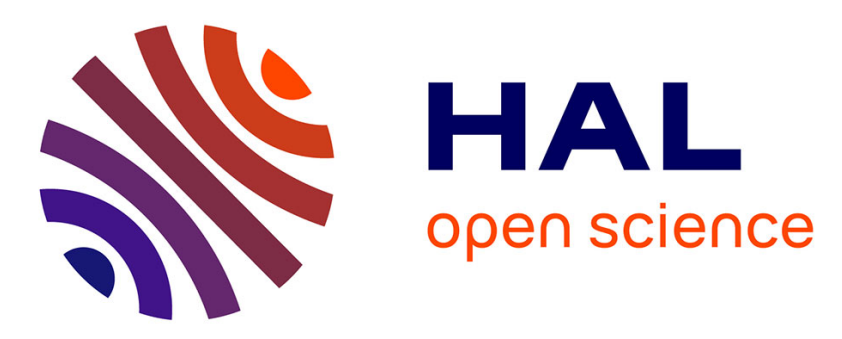

\title{
A UMI3D-based Interactions Analytics System for XR Devices and Interaction Techniques
}

\author{
Julien Casarin, Antoine Ladrech, Tristan Tchilinguirian, Dominique \\ Bechmann
}

\section{- To cite this version:}

Julien Casarin, Antoine Ladrech, Tristan Tchilinguirian, Dominique Bechmann. A UMI3D-based Interactions Analytics System for XR Devices and Interaction Techniques. IEEE VR Conferences on Virtual reality and 3D User Interface, 2019, Osaka, Japan. 10.1109/VR.2019.8797958 . hal-03238127

\section{HAL Id: hal-03238127 \\ https://hal.science/hal-03238127}

Submitted on 26 May 2021

HAL is a multi-disciplinary open access archive for the deposit and dissemination of scientific research documents, whether they are published or not. The documents may come from teaching and research institutions in France or abroad, or from public or private research centers.
L'archive ouverte pluridisciplinaire HAL, est destinée au dépôt et à la diffusion de documents scientifiques de niveau recherche, publiés ou non, émanant des établissements d'enseignement et de recherche français ou étrangers, des laboratoires publics ou privés. 


\section{A UMI3D-based Interactions Analytics System for XR Devices and Interaction Techniques}

\author{
Julien Casarin* \\ Gfi Informatique
}

\author{
Antoine Ladrech \\ Gfi Informatique
}

\author{
Tristan Tchilinguirian \\ Gfi Informatique
}

\author{
Dominique Bechmann \\ University of Strasbourg
}

\begin{abstract}
In this paper we present an interaction analytics system we are working on. With this system we intend to simplify the evaluation and classification of eXtented Reality devices and interaction techniques. Our final objective is to release it as an open Cloud platform that will allow researchers to compare their respective results in the field of Human-Computer Interaction with ease. To achieve this, we use the UMI3D exchange protocol to design device-independent 3D environments, and a Cloud analytics platform which stores experimental raw data from these environments as well as from the different devices. Finally, we present our first results using the platform to compute statistical analyzes and create dashboards comparing a set of devices and interaction techniques.
\end{abstract}

Index Terms: H.3.5 [Online Information Services]: Data sharing; I.3.6 [Methodology and Techniques]: Interaction techniques; H.5.2 [User Interfaces]: Evaluation/methodology; H.5.1 [Multimedia Information Systems]: Artificial, augmented, and virtual realities

\section{INTRODUCTION}

3D interaction devices multiply and people have tried to evaluate their newly designed products or interaction techniques to ensure that they are convenient to use. What is generally done is a comparison between several devices and an analysis of the relative performances according to different metrics. To do so, people usually organize test sessions where a group of users performs a sequence of elementary tasks on each device.

So far, people have mostly evaluated one specific device or technique at a time, that they compared to other reference devices $[1,5,6]$. However, it is difficult to program a test application that is universally supported by all existing devices and those that are to come. Thus, programmers have re-developed their applications over and over again and similar test sessions have been done on the same device at several occasions without saving any data or result.

Recently [3], Casarin et al. introduced the UMI3D protocol which permits the design of $3 \mathrm{D}$ media regardless of the user devices. In the remainder of this paper, we propose to combine the UMI3D protocol with a cloud-based analytics platform to build a standard evaluation and classification protocol of eXtended Reality (XR) devices and interaction techniques. Then, we describe the procedure we have followed to use this protocol for the creation of a comparative dashboards of devices' performances for given UMI3D environments.

\section{UMI3D}

The UMI3D data exchange protocol introduced by Casarin et al. in [3] allows the interaction with a 3D media regardless of user devices. To achieve this, a finite set of objects called Interaction Blocks are exchanged in real time between a 3D environment and some connected clients. These Interaction Blocks are used as an interaction-based abstraction layer.

*e-mail: julien.casarin@gfi.fr

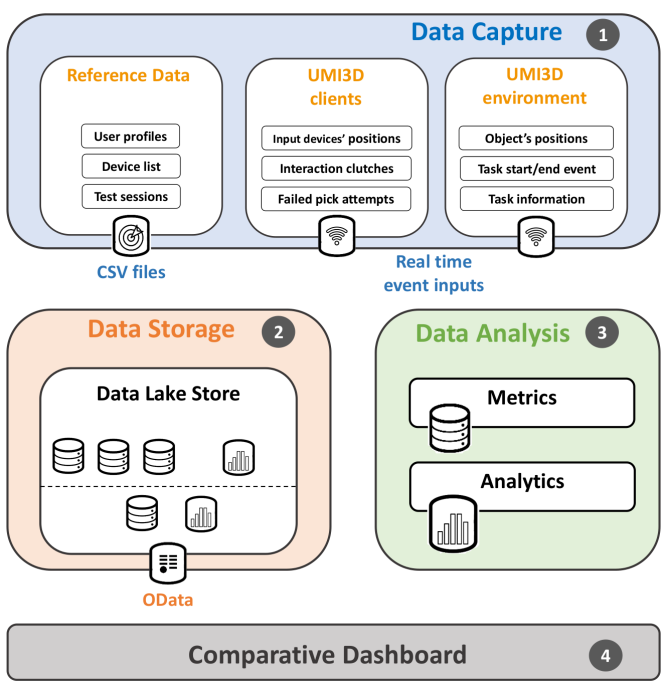

Figure 1: DaaS' place in the device classification project

According to [3], the only requirement to support new devices on existing UMI3D environments is the implementation of a dedicated client for each of these devices. The purpose of a UMI3D client is to connect the device to the 3D environments and to interpret the distributed Interaction Blocks depending on the device's inputs. We believe this property very interesting in order to compare different devices in the execution of a given set of tasks. Indeed, a UMI3D environment can easily be shared to any researcher working on new interaction techniques. In this case, it would be possible to reproduce exactly the same conditions as in previous experiments by running the same application.

\section{COMBINING UMI3D WITH AN ANALYTICS PLATFORM}

\subsection{Used analytics platform}

In order to let researchers compare their results to existing test sessions and compute new analytics on the stored data, we needed a scalable storage system for all the experimental raw data generated over time (like the position and rotation of the tracked objects at every frame). To achieve that, we use a cloud analytics platform developped by oyr organization, DaaS. Among all the capabilities and feature of this platform, we describe here only those that are relevant to our study:

- A great amount of data can be stored in a Data Lake (2 in Fig. 1) as the platform uses the Hadoop distributed file system [2]. This allows us to store data that can be accessed at a later date for further analysis.

- DaaS also provides a way to create and execute U-SQL queries (3 in Fig. 1) (a Big-Data-oriented query language of the Azure Data Lake Analytics service) in which it was 
made possible to integrate $\mathrm{R}$ code (a broadly used programming language for statistical computing [4]). Those requests apply on any of the data stored in the Data Lake to process it.

- It is also possible for external applications to gather data from the platform to display it or perform additional computations through the OData protocol. (4 in Fig. 1)

\subsection{Crossing UMI3D environment's and client's data}

In order to evaluate and classify UMI3D clients, we need to cross data sent in real time from both the UMI3D environment (such as the successive positions of the object being manipulated) and the UMI3D client (all the data concerning the device itself). To synchronize the data between the client and the environment (i.e. to know which client's data is related to which environment's data), we added timestamps to the real-time data.

In addition to the environment's and client's real-time data, several reference CSV files (represented by the 'Reference Data' sections in Fig. 1) are manually updated to keep track of all the entities relevant to the classification. They contain information about the test sessions, the users and the devices.

Finally, additional data concerning the parameters of the tasks and their starting time are sent once at their start, and their ending time is sent when they are completed.

\section{FIRST RESULTS}

\subsection{From choosing tasks to computing metrics and analyses}

We focused our study on three of the most common types of tasks in 3D interaction: selection, translation and rotation of an object in a 3D environment. For this purpose, we have created a UMI3D environment that can generate reference tasks of each type. Once the application started, any UMI3D client can connect and start completing the tasks as they appear. We also added parameters to customize the tasks, such as type, difficulty and number of degrees of freedom (only for translation). A time limit was also added to determine if the user successfully passed the tasks or not, and prevent him from being stuck on a single one. For each task, we have arbitrarily selected and computed metrics found in diverse papers. Those chosen are elementary and interpretable at first sight so that they fit with the visual aspect of dashboards. We also wanted them to cover general aspects of the tasks' completion, such as speed or precision. Thus, we selected metrics such as success rate, completion time (all tasks) or final distance (translation tasks only).

Then, we implemented different queries to compute those metrics for each task and other queries to perform statistical analyses of these metrics. First, basic values such as averages, medians or standard deviations were computed since they give a visual approach of the tasks results as they can easily be plotted in graphs. However, more advanced statistical tests have to be performed to draw conclusions from the data. To demonstrate the feasibility of implementing such an analysis, we integrated on the DaaS platform a R script of our own that uses the ANOVA study. The platform allowed us to store different values from the study such as Fisher's exact test results, the $\mathrm{p}$-values or the test power values.

\subsection{Creating graphs and dashboards}

The ultimate purpose of our system is the creation of dashboards where to display the results of those statistical analyses. to give a global and comparative overview of the devices' performances on the tasks. The classification dashboards are accessible through a web application. A huge advantage of this procedure is that the application does not compute anything on its own, apart from what is necessary to the visualization of the data. It considerably lightens the dashboards which increases their responsivity. Moreover, the sections are individually interactive, allowing the user to sort out

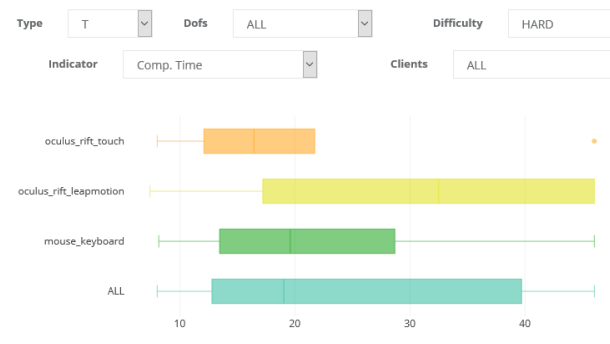

Figure 2: Boxplots of the completion time for translation tasks with a high difficulty

only the tasks he is interested in. He can indeed specify the type, difficulty and amount of degrees of freedom of the tasks, as well as the devices and metrics he wants to display. Fig. 2 shows one of the graphs we have implemented and integrated to our dashboard.

\section{Conclusion}

In this paper, we have presented an analytics system that simplifies the comparison of XR devices and interaction techniques. Yet, it is still a work in progress and is only adapted for a single researcher or team. A bigger challenge would be to allow the comparison between new experimentations and data already collected by other researchers around the world. Sharing the data between several actors on the DaaS platform does not add any technical limitations since the data is already stored in the cloud. Indeed, the technology used for the analytics platform is fully scalable and permits the storage of a huge quantity of data at a very high throughput.

However, there is no way yet to guarantee the relevance and integrity of the data, which researchers might not trust. Part of it comes from the inability to store elements about the context of the data collection. This is why we assume that further work needs to be done in that regard, as for instance the establishment of a complementary data structure. This data structure could contain among others a description of the experimental setup, and a reference to the article related to a particular data set. An other idea is the creation of a peer-review system to ensure the relevance of the data before it is shared.

\section{REFERENCES}

[1] F. Bérard, J. Ip, M. Benovoy, D. El-Shimy, J. R. Blum, and J. R. Cooperstock. Did "minority report" get it wrong? superiority of the mouse over $3 \mathrm{~d}$ input devices in a $3 \mathrm{~d}$ placement task. In T. Gross, J. Gulliksen, P. Kotzé, L. Oestreicher, P. Palanque, R. O. Prates, and M. Winckler, eds., Human-Computer Interaction - INTERACT 2009, pp. 400-414. Springer Berlin Heidelberg, Berlin, Heidelberg, 2009.

[2] D. Borthakur. The hadoop distributed file system: Architecture and design. Hadoop Project Website, 11(2007):21, 2007.

[3] J. Casarin, D. Bechmann, and M. Keller. A unified model for interaction in $3 \mathrm{~d}$ environment. In Proceedings of the 23rd ACM Symposium on Virtual Reality Software and Technology, VRST '17, pp. 23:1-23:7. ACM, New York, NY, USA, 2017. doi: 10.1145/3139131.3139140

[4] R. Ihaka and R. Gentleman. R: a language for data analysis and graphics. Journal of computational and graphical statistics, 5(3):299-314, 1996.

[5] M. Ortega and L. Nigay. Airmouse: Finger gesture for $2 d$ and $3 d$ interaction. In T. Gross, J. Gulliksen, P. Kotzé, L. Oestreicher, P. Palanque, R. O. Prates, and M. Winckler, eds., Human-Computer Interaction - INTERACT 2009, pp. 214-227. Springer Berlin Heidelberg, Berlin, Heidelberg, 2009.

[6] M. Raynal, E. Dubois, and B. Schmitt. Towards unification for pointing task evaluation in $3 \mathrm{~d}$ desktop virtual environment. In A. Holzinger, M. Ziefle, M. Hitz, and M. Debevc, eds., Human Factors in Computing and Informatics, pp. 562-580. Springer Berlin Heidelberg, Berlin, Heidelberg, 2013. 